\title{
Single-Spin Magnetomechanics with Levitated Micromagnets
}

\author{
J. Gieseler $\odot,{ }^{1,{ }^{*}, \dagger}$ A. Kabcenell $\odot,{ }^{1}$ E. Rosenfeld,,${ }^{1}$ J. D. Schaefer $\odot,{ }^{1}$ A. Safira, ${ }^{1}$ M. J. A. Schuetz,${ }^{1}$ \\ C. Gonzalez-Ballestero $\odot^{2,3}$ C. C. Rusconi, ${ }^{4}$ O. Romero-Isart $\odot,{ }^{2,3}$ and M. D. Lukin ${ }^{1}$ \\ ${ }^{1}$ Physics Department, Harvard University, Cambridge, Massachusetts 02138, USA \\ ${ }^{2}$ Institute for Quantum Optics and Quantum Information of the Austrian Academy of sciences, A-6020 Innsbruck, Austria \\ ${ }^{3}$ Institute for Theoretical Physics, University of Innsbruck, A-6020 Innsbruck, Austria \\ ${ }^{4}$ Max-Planck-Institut für Quantenoptik, Hans-Kopfermann-Strasse 1, 85748 Garching, Germany
}

(Received 22 December 2019; accepted 16 March 2020; published 24 April 2020)

\begin{abstract}
We demonstrate a new mechanical transduction platform for individual spin qubits. In our approach, single micromagnets are trapped using a type-II superconductor in proximity of spin qubits, enabling direct magnetic coupling between the two systems. Controlling the distance between the magnet and the superconductor during cooldown, we demonstrate three-dimensional trapping with quality factors around $1 \times 10^{6}$ and $\mathrm{kHz}$ trapping frequencies. We further exploit the large magnetic moment to mass ratio of this mechanical oscillator to couple its motion to the spin degrees of freedom of an individual nitrogen vacancy center in diamond. Our approach provides a new path towards interfacing individual spin qubits with mechanical motion for testing quantum mechanics with mesoscopic objects, realization of quantum networks, and ultrasensitive metrology.
\end{abstract}

DOI: 10.1103/PhysRevLett.124.163604

Realizing coherent coupling between individual spin degrees of freedom and massive mechanical modes is an outstanding challenge in quantum science and engineering. The spin's strong quantum nonlinearity facilitates preparation of macroscopic quantum states of motion [1], while the mechanical mode can mediate effective spin-spin interactions between distant spin qubits [2]. This enables applications in quantum information processing [3], sensing [4-6], and fundamental physics [7,8]. One particularly promising approach is to engineer a strong spin-mechanical coupling via magnetic field gradients [9-13]. Achieving strong spin-resonator coupling requires a combination of high quality mechanical resonators, strong magnetic field gradients, and spin qubits with very long spin coherence times, such that the coupling exceeds the decoherence rates of the two subsystems.

In this Letter, we propose and demonstrate a new platform for strong spin-mechanical coupling based on levitated microscopic magnets coupled to the electronic ground state of a single nitrogen vacancy (NV) center in diamond (Fig. 1). The key idea is to utilize a levitated magnet that is localized in free space by electromagnetic fields. In such a system, dissipation is minimized since there is no direct contact with the environment.

Published by the American Physical Society under the terms of the Creative Commons Attribution 4.0 International license. Further distribution of this work must maintain attribution to the author(s) and the published article's title, journal citation, and DOI.
Specifically, we make use of a levitating mechanical resonator based on magnetostatic fields. This approach not only avoids clamping losses, but also circumvents photon recoil and heating associated with optical levitation [14-18] and is therefore predicted to yield large mechanical quality factors $[19,20]$. In addition, the levitated magnet naturally generates the strong magnetic field gradient that is required for spin-mechanical coupling. We specifically demonstrate the coupling to an individual NV center, one of the most studied color centers in diamond [21]. Besides optical initialization and readout, the NV center features long coherence times, which makes it an attractive candidate for scalable quantum networks in the solid state [3], quantum sensing [22-24], and quantum communication [25].

So far, experiments with superconducting levitation have been limited to millimeter-scale magnets [26-29]. A recent experiment [30] demonstrated superconducting levitation of micromagnets, but without spin-mechanical coupling and with much lower frequencies and $Q$ factors than shown here. Levitated spin-mechanical systems in which the spin is hosted inside the resonator have been implemented with nanodiamonds containing NV center defects trapped in optical tweezers [31,32], Paul traps [33-35], and magnetogravitational traps [36,37]. Nonetheless, the challenge remains to integrate these systems with strong magnetic field gradients, long coherence NV centers and operation under ultrahigh vacuum conditions. Our approach fulfills all these criteria simultaneously (Fig. 1).

Levitation of micromagnets.-We levitate single hard micromagnets with a thin film of the type-II superconductor 
(a)

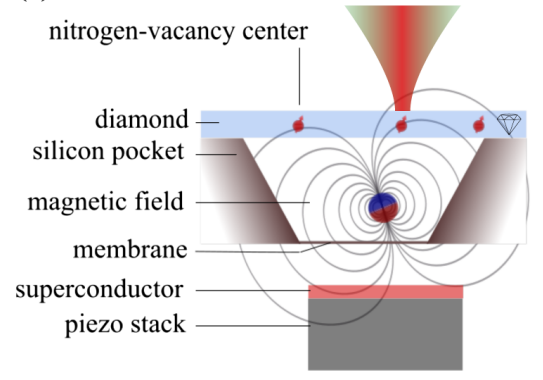

(b)

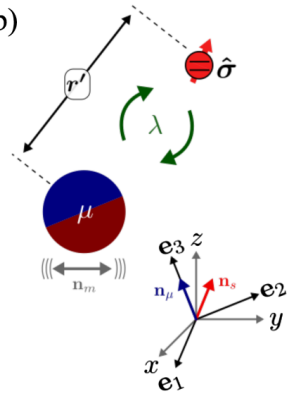

FIG. 1. Concept of the experiment. (a) Individual microscopic hard ferromagnets are isolated in microfabricated pockets and levitated with a type II superconductor, exploiting its flux trapping properties. The magnet's stray field allows for efficient coupling between the magnet's motional degrees of freedom and bulk nitrogen vacancy centers in the nearby diamond, which generally feature long spin coherence times. (b) The coupling $\lambda$ depends on the relative orientation of the NV center $\mathbf{n}_{s}$, the magnetic moment $\mathbf{n}_{\mu}$, the direction of motion of the magnet $\mathbf{n}_{m}$, the distance between the magnet and the NV center $r^{\prime}$, the magnet radius $a$, and the frequencies of the motion.

(sc) YBCO (cf. Fig. 1). Since we do not apply magnetic fields, the micromagnet is the only magnetic field source. Thus, magnetic flux through the $\mathrm{YBCO}$ is determined only by the magnet-YBCO distance $h_{\text {cool }}$ and the magnet orientation $\theta_{\text {cool }}$. After cooling YBCO below its critical temperature $T_{c} \approx 90 \mathrm{~K}$, it becomes superconducting and traps the magnetic flux that penetrates it. Consequently, below $T_{c}$, motion of the magnet induces currents in the superconductor that counteract changes in the magnetic field. This allows for stable 3D trapping using a procedure illustrated in Fig. 2(a), with levitation height $\left(h_{\text {lev }}\right)$ and trapping frequencies $\omega_{j}(j=x, y, z)$ depending on the conditions during cooldown [38].

We observe the levitated magnet through a microscope objective that is positioned outside the vacuum chamber, and record its motion with a fast camera [Fig. 2(b)]. From the video frames, we extract time traces of the particle's center-of-mass position in the camera coordinate system $\left[x_{c}(t), y_{c}(t)\right]$ and calculate their power spectral densities (PSD). The distinct peaks in the PSDs correspond to the three center-of-mass modes $\omega_{j} / 2 \pi$ [Fig. 2(c)].

Figure 2(d) displays the center-of-mass frequencies as a function of the normalized levitation height $\bar{h}_{\mathrm{lev}}=$ $h_{\text {lev }} / a$ for two particles with radius $a_{1}=23.2 \pm 0.7$ and $a_{2}=15.5 \pm 0.3 \mu \mathrm{m}$, respectively. The lines are a fit to a power law $f\left(\bar{h}_{\text {lev }}\right)=f_{\max } \bar{h}_{\text {lev }}^{-\beta}$, where $f_{\max }$ is the frequency in the limit when the gap between the particle and the superconductor vanishes. In our experiment, we find $f_{\max }=(2.3 \pm 0.4,2.4 \pm 0.4,5.6 \pm 1.0) \mathrm{kHz}$ and $\beta=(1.9 \pm 0.1,2.1 \pm 0.1,2.0 \pm 0.1)$ for particle 1 and $f_{\max }=(8.8 \pm 1.1,9.5 \pm 1.1,25.2 \pm 3.3) \mathrm{kHz}$ and $\beta=(2.1 \pm 0.1,2.1 \pm 0.1,2.3 \pm 0.1)$ for particle 2 , which is in good agreement with the expected value of $\beta=2.5$ (a)

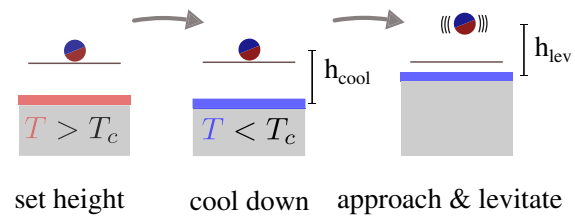

(b)
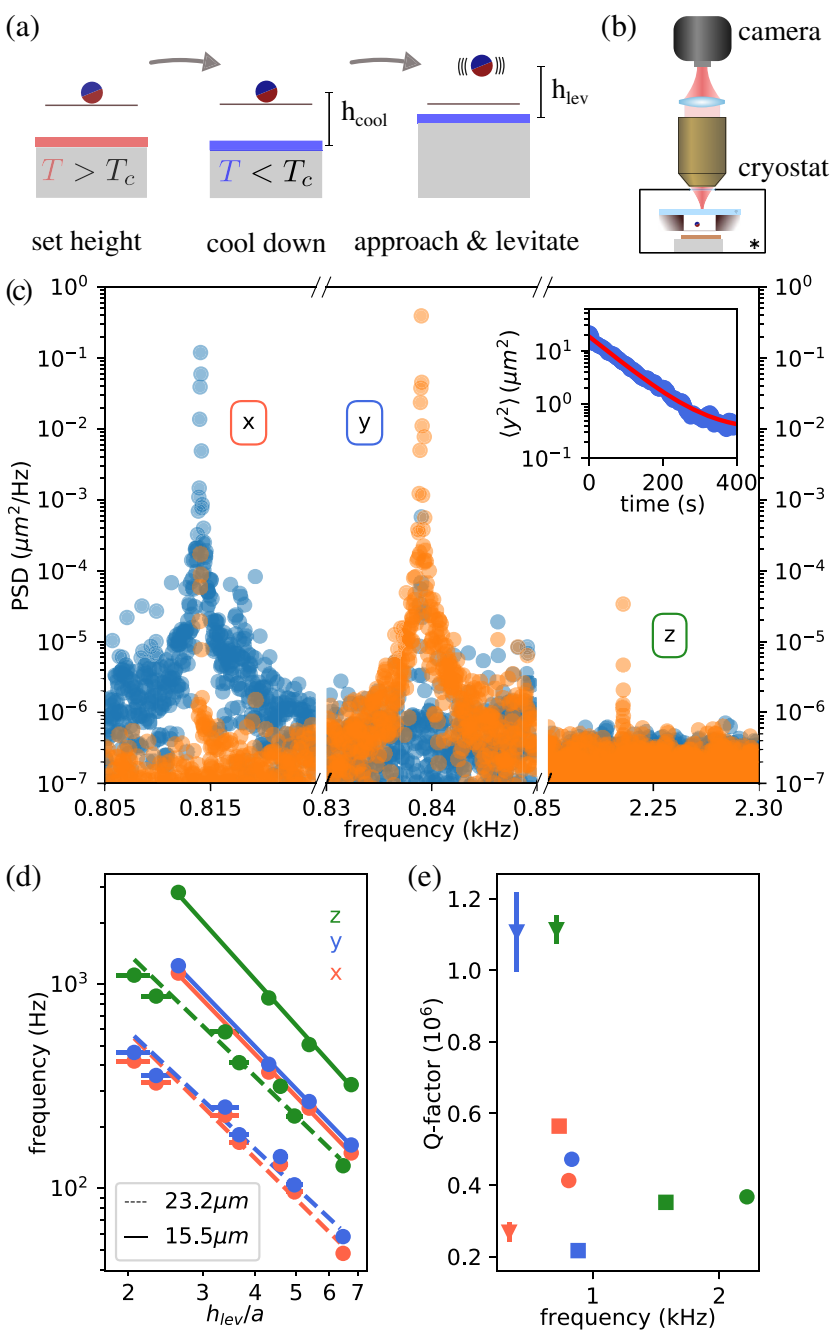

FIG. 2. Mechanical properties of levitated micromagnets. (a) To levitate the magnets, we adjust the magnet-sc distance above the critical temperature $T_{c}$. Then we cool the sc below $T_{c}$ to freeze in the magnetic flux from the magnet. After cooldown, we reduce the distance until the magnet begins to levitate. (b) We observe the magnet motion through a microscope objective and record its motion with a video camera. (c) Power spectral density extracted from video analysis of the magnet motion in the vertical (horizontal) direction, corresponding to the data shown as circles in (e). The inset shows a typical ringdown measurement of the $y$ mode $\left(\omega_{y}=0.839 \mathrm{kHz}\right)$ from which we extract the $Q$ factor. (d) Frequencies of center-of-mass motion as a function of the levitation height normalized to magnet radius. Dashed (solid) lines show the three center-of-mass modes for magnet 1 (2). (e) $Q$ factor as a function of trap frequency for magnet 2. Each symbol corresponds to a different levitation experiment and the different colors correspond to the three different center-of-mass modes.

from a simple dipole model [38]. The measured center-ofmass frequencies are comparable to those achieved with optical levitation [16,17] and significantly exceed motional frequencies in Paul traps [41] and magnetogravitational traps [36,37]. However, the observed dependence of the 
maximum frequency on the particle radius is stronger than the dipole model's prediction of $f_{\max }^{\mathrm{dp}} \propto 1 / a$. We attribute this to the multidomain nature of our particles and note that spherical particles as large as $a \sim 1 \mu \mathrm{m}$ can be single domain [42].

Figure 2(e) shows the $Q$ factors of magnet 1 for three different levitation heights for all three translational modes. We measure the dissipation with ring down measurements, exciting one mode with an ac magnetic field and observing its energy decay [Fig. 2(c), inset]. From an exponential fit we extract the decay time $1 / \gamma_{j}$ and the $Q$ factor $Q_{j}=\omega_{j} / \gamma_{j}$ for each mode. The measured $Q$ factors are around $1 \times 10^{6}$ and depend only weakly on the trapping frequencies and thus on the levitation height. Air damping can be ruled out at our experimental conditions with pressures below $10^{-5} \mathrm{mBar}$ and the most likely source of dissipation is the magnet-superconductor interaction [38]. Note that, even though this $Q$ factor is somewhat lower than what has been demonstrated with nonmagnetic optically levitated [18] and nanofabricated mechanical resonators [43-46], it represents the state of the art for magnetized resonators $[47,48]$ and the ultimate limit, in particular for magnets with $a<1 \mu \mathrm{m}$, is still an open question.

Coupling to NV center.-Next, we demonstrate coupling the motion of a levitated micromagnet to the electronic spin associated with an individual negatively charged NV center. In our sample, NV centers are hosted inside a diamond slab and implanted $d_{\text {impl }} \sim 15 \mathrm{~nm}$ below the diamond surface. The diamond replaces the glass slide of the previous experiment and is placed across the pocket that contains the magnet. The pocket is $\sim 80 \mu \mathrm{m}$ deep and the magnet radius is $a_{3}=15.1 \pm 0.1 \mu \mathrm{m}$. We levitate the magnet $z_{m d}=44 \pm 5 \mu \mathrm{m}$ below the diamond using the same method as before. The NV center is located at $\left(x_{d}, y_{d}\right)=(83,29) \pm 5 \mu \mathrm{m}$ with respect to the magnet center such that the magnet-NV distance is $\left|\mathbf{r}^{\prime}\right|=$ $\sqrt{\left(z_{m d}+a_{3}\right)^{2}+x_{d}^{2}+y_{d}^{2}}=99 \pm 5 \mu \mathrm{m}$. The NV center's electronic ground state has spin $S=1$ with the lowerenergy $\left|m_{s}=0\right\rangle$ level separated from the $\left|m_{s}= \pm 1\right\rangle$ levels by a zero-field splitting $D_{\mathrm{zf}} /(2 \pi) \approx 2.87 \mathrm{GHz}$ and its symmetry axes $\mathbf{n}_{\mathrm{s}}$ aligned along one of four crystallographic orientations set by the tetrahedral symmetry of the diamond lattice. A microwave (MW) signal at the transition frequency $\omega_{\mathrm{MW}}$, drives the transition $\left|m_{s}=0\right\rangle \rightarrow$ $\left|m_{s}= \pm 1\right\rangle$ which results in a decrease of the photoluminescence (PL) signal. The spin-dependent PL of the NV defect is due to a nonradiative intersystem crossing decay pathway, which also allows for efficient spin polarization in the $\left|m_{s}=0\right\rangle$ spin sublevel through optical pumping [49]. The magnetic field dependent PL can therefore be used to optically detect magnetic fields [50], which is used to sense the motion of the magnet [Figs. 3(a) and 3(b)].
Figure 3(c) shows the optically detected magnetic resonance (ODMR) spectrum of the NV center with a fit to a Lorentzian corresponding to the $|+1\rangle$ transition. The spectrum is measured with a home-built fluorescence microscope that we integrated with the cryostat [Fig. 3(a)]. In the presence of a microwave tone, the spin-mechanical coupling $\lambda_{g}$ causes a variation in PL, since a displacement $x$ of the magnet shifts the electron spin resonance by $\delta \omega_{\mathrm{NV}}=\left(\lambda_{g} / x_{\mathrm{zp}}\right) x$, where $x_{\mathrm{zp}}=\sqrt{\hbar / 2 m \omega_{x}}$ is the zero point motion. To measure the magnet's motion with the $\mathrm{NV}$, we excite one of its modes with a broadband fluctuating magnetic field, driving it into a quasithermal state and allowing us to observe it as a peak in the PSD of the NV PL counts [Fig. 3(f)]. We confirm that the peak is due to the moving magnet with the camera [Fig. 3(d)]. The camera and NV measurements are taken sequentially. Notably, we observe a small systematic frequency shift of $\approx 1 \mathrm{~Hz}$ between the $\mathrm{NV}$ and camera measurements, which is likely due to the laser turned on during the NV measurement.

The mean spectral power in the NV peak is $\left\langle c_{\mathrm{NV}}^{2}\right\rangle=$ $s^{2}\left\langle\delta \omega_{\mathrm{NV}}^{2}\right\rangle$, where $s$ is the slope of the ODMR signal at the microwave frequency, which we measure by applying a calibration tone to the microwave signal. The mean spectral power in the camera measurement $\left\langle x^{2}\right\rangle$ allows us then to extract the spin-mechanical coupling as $\lambda_{g}=$ $x_{\mathrm{zp}} \sqrt{\left\langle\delta \omega_{\mathrm{NV}}^{2}\right\rangle /\left\langle x^{2}\right\rangle}$. To measure the coupling and confirm the thermal character of the driven mode, we consider the area under the PSD integrated over a time interval much shorter than $1 / \gamma$, and construct its distribution over repeated measurements. For both the camera and the NV measurements, the distribution agrees with an exponential distribution $P(E)=\beta_{E} \exp \left(-\beta_{E} E\right)$, where $\beta_{E}$ is the inverse of the variances $\left\langle x^{2}\right\rangle$ and $\left\langle\delta \omega_{\mathrm{NV}}\right\rangle$ [Figs. 3(e) and 3(g)]. The resulting coupling strength is $48 \pm 2 \mathrm{mHz}$, in satisfactory agreement with the theoretical value for the gradient coupling to a dipole $\lambda_{g}=\gamma_{e}\left(\mu_{0} \rho_{\mu} a^{3} / r^{\prime 4}\right) x_{\mathrm{zp}} f_{g}(\theta)=2 \pi \times$ (18 \pm 3$) \mathrm{mHz}_{g}(\theta)$ [Fig. 4(b)]. Here, $f_{g}(\theta) \sim 1$ depends on the relative position and orientation of the $\mathrm{NV}$ center and the magnet.

Discussion.-We now discuss the prospects of using this system to achieve strong coupling. The minimal NV-magnet separation $d_{q}^{\text {min }}=\left|\mathbf{r}^{\prime}\right|-a$ is given by the $\mathrm{NV}$-implantation depth and the onset of strong attractive surface forces that will make the magnet stick to the diamond surface. Assuming that the frequency scales as $\omega_{j} / 2 \pi=c_{f} a^{-n}$, the radius $a=(n+3) /(5-n) d_{q}^{\min }$ yields the maximum gradient coupling for a dipolar particle. A conservative gap $d_{q}^{\min }=0.25 \mu \mathrm{m}, c_{f}=15 \mathrm{kHz} \mu \mathrm{m}$, corresponding to our observations in Fig. 2, and $n=1$ for the dipole model, results in $a=0.25 \mu \mathrm{m}$ and $\lambda_{g} / 2 \pi \sim$ $2.6 \mathrm{kHz}$ (Fig. 4). Since the motional frequency can be reduced by adjusting the levitation height, one can even reach the elusive ultrastrong coupling regime $\lambda_{g}>\omega_{j}$ [51]. 
(a)

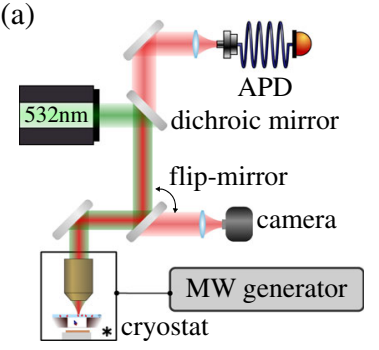

(b)

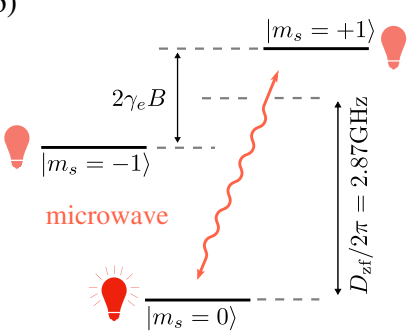

(d)

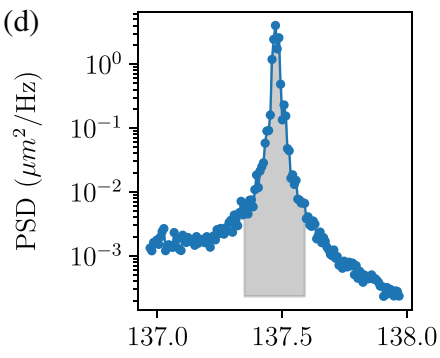

frequency $(\mathrm{Hz})$

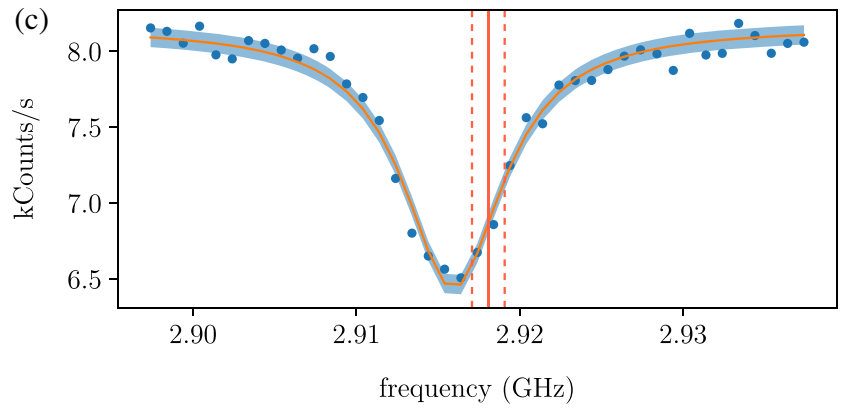

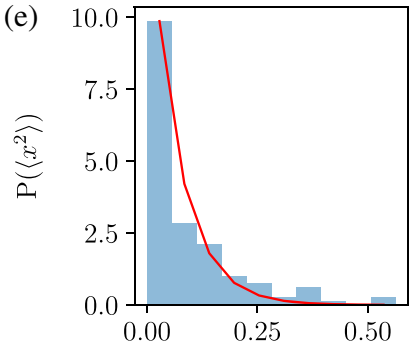

$\left\langle x^{2}\right\rangle\left(\mu m^{2}\right)$

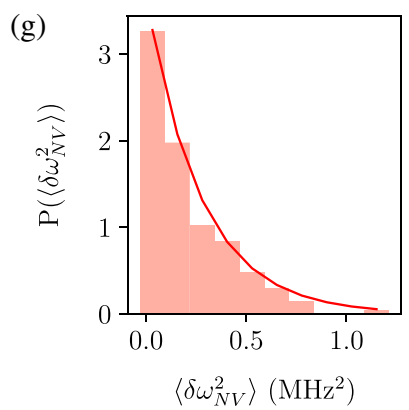

FIG. 3. Measurement of spin-motional coupling. (a) Schematic of the experiment. (b) Nitrogen-vacancy ground state spin levels. Resonant microwaves drive transitions between the $m_{s}=0$ and $m_{s}= \pm 1$ states. The transition frequency depends on the magnetic field, which depends on the magnet-NV distance. The $m_{s}=0$ is brighter than the $m_{s}= \pm 1$ states. Hence, for near-resonant MW driving, the fluorescence intensity depends on the magnet position. (c) ODMR spectrum. The magnet motion is measured by recording a time trace of the fluorescence photons while applying a MW tone at the steepest slope of the transition (2.918 GHz) (solid red line). We measure the slope by frequency modulating the MW tone (red dashed lines). (d) Power spectral density of the video time trace and (f) fluorescence time trace. The gray area corresponds to the numerical integration of the variance $\left\langle x^{2}\right\rangle,\left\langle c_{\mathrm{NV}}^{2}\right\rangle$, and $\left\langle c_{\text {cal }}^{2}\right\rangle$. The second sharp peak is the calibration peak due to the MW frequency modulation. The black dashed line represents the photon shot noise. Histograms of the variances reveal the thermal character of the motional state during both the (e) camera and (g) NV measurement which we fit (red line) to extract the variances $\left\langle x^{2}\right\rangle$ and $\left\langle\delta \omega_{\mathrm{NV}}^{2}\right\rangle$, respectively.

The cooperativity $C=\lambda^{2} Q \tilde{T}_{2} \hbar /\left(2 \pi k_{B} T\right)>1$ marks the onset of coherent quantum effects in a coupled spinphonon system. With a mechanical $Q$ factor of $10^{8}$, which has been demonstrated in levitated systems [52], the coupling exceeds the thermal decoherence rate $\Gamma_{\mathrm{th}} / 2 \pi=$ $k_{B} T /(2 \pi \hbar Q)=0.8 \mathrm{kHz}$ at $T=4 \mathrm{~K}$. NV centers in bulk diamond, such as the sample used in our experiment, can have up to second-long extended coherence times $\tilde{T}_{2}$ at these temperatures using spin manipulation such as multipulse dynamical decoupling sequences limited only by pulse errors [53,54]. The minimum spin manipulation frequency in such sequences is typically a few $\mathrm{kHz}$ for bulk diamond NV centers, which is within reach for the mechanical frequencies in our current geometry. Hence, this system can reach the high cooperativity $(C>1)$ and even the strong coupling regime $\left(\lambda>2 \pi / \tilde{T}_{2}, \Gamma_{\text {th }}\right)$. Such a strong coupling enables ground-state cooling, quantum-byquantum generation of arbitrary states of motion [9], and spin-spin entanglement [2].

Besides the translational degrees of freedom, levitated particles are free to rotate. For the hard magnets used in our experiment, the anisotropy energy strongly couples the particle orientation to the magnetization axis. The coupling leads to hybrid magnetorotational modes, which
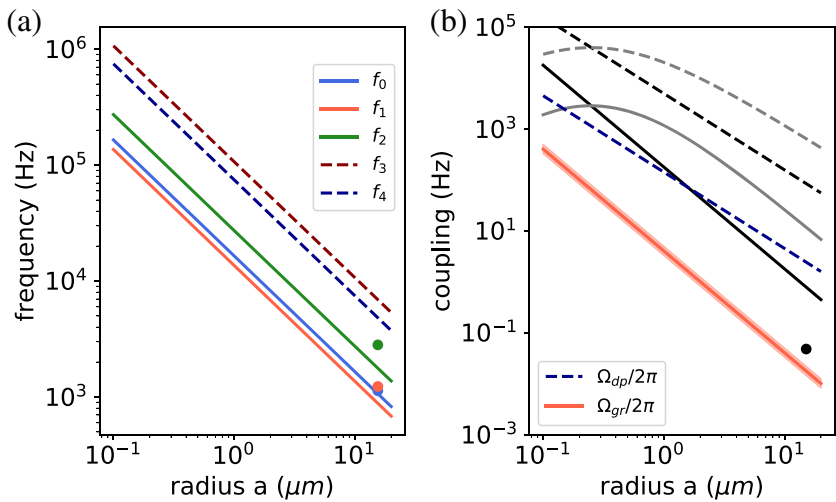

FIG. 4. Prospect for frequencies and couplings. (a) Mode frequency following dipole model [39] for $h_{\mathrm{lev}} / a=3$. The lowest (solid) lying modes correspond to the center of mass and the highest (dashed) lying modes to rotational motion. Data points are the experimental frequencies for particle 1 Fig. 2(d). $\mathrm{MHz}$ frequencies are predicted for sub- $\mu \mathrm{m}$ particles. (b) Gradient (solid) and dipole-dipole (dashed) couplings, respectively. Straight lines show couplings for constant gap-particle size ratio $\bar{d}=d / a=5.5$ (color) and $\vec{d}=1$ (black). Curved lines (gray) show coupling for constant gap $d=250 \mathrm{~nm}$ with a maximum coupling at $a=d$. The black data point is the experimental gradient coupling for particle 3 . 
correspond to a librational mode at frequency $\omega_{l}=\sqrt{\omega_{L} \omega_{I}}$ that precesses around the local magnetic field $\mathbf{B}_{0}$ at the Einstein-de Haas frequency $\omega_{I}=\rho_{\mu} V_{\mathrm{mag}} /\left(I_{0} \gamma_{0}\right)$ due to the intrinsic spin angular momentum of the polarized electrons in the magnet. Here, $\mathbf{B}_{0}$ is the sum of the field due to the sc and additional external fields and $I_{0}=2 \rho_{m} V_{\mathrm{mag}} a^{2} / 5$ is the moment of inertia, $V_{\text {mag }}$ being the volume of the magnet and $\rho_{m}\left(\rho_{\mu}\right)$ its mass (spin) density. Since the Larmor frequency $\omega_{L}=\gamma_{0} B_{0}, \omega_{l}$ is higher than the translational mode frequencies even without additional fields and can be tuned with moderate magnetic fields $\sim 10 \mathrm{mT}$ to $\mathrm{MHz}$ frequencies. The high frequencies of the librational modes make them inaccessible to our current detection based on video analysis and dc magnetometry. Future work will explore these modes using optical interferometry and SQUID [55] or NV-ac magnetometry [4,41]. The rotational modes couple to the $\mathrm{NV}$ center with a dipole-dipole coupling $\lambda_{\mathrm{dp}}=\gamma_{e} \mu_{0} m_{z p}\left(a^{3} / r^{\prime 3}\right) f_{\mathrm{dp}}(\theta)$, where $f_{\mathrm{dp}}(\theta) \sim 1$ depends on the relative position and orientation of the NV center and the magnet, and $m_{\mathrm{zp}}=\sqrt{\hbar \gamma_{e} \rho_{\mu} / 2 V_{\mathrm{mag}}}$ is the zero point magnetization of the Kittel magnon [56]. The weaker distance dependence yields $\lambda_{\mathrm{dp}}=0.4 \mathrm{kHz}$ for a $a=5 \mu \mathrm{m}$ magnet at $5 \mu \mathrm{m}$ distance from the NV center [Fig. 4(b)]. This parameter regime is readily accessible with the experimental approach presented here, and it is sufficient to probabilistically cool the librational mode near its ground state [57].

Outlook.-These considerations indicate that our approach is a promising platform for quantum nanomechanics. Our experimental technique also allows us to achieve levitation with the superconductor in the Meissner state, and thus presents a path forward to observe precession due to the intrinsic spin angular momentum of the magnet with applications in highly sensitive magnetometry [58]. Since our mechanical resonator is all magnetic, we maximize the spin-to-mass ratio $\rho_{\mu} / \sqrt{\rho_{m}}$ for a given magnetic material, which maximizes the spin-mechanical coupling. This leads to strong spin-mechanical coupling even for moderate experimental parameters. In addition, this system features libration modes, which are expected to reach an unprecedented spin-mechanical parameter regime even for magnets with $a \sim 5 \mu \mathrm{m}$. Magnets of this size can be levitated with the experimental technique introduced here. The combination of high mechanical $Q$ factor, strong spin-mechanical coupling, and long spin coherence is key for a range of applications such as magnetometers, accelerometers, and gyroscopes [55], where the magnet is the sensor which is read out through the NV center [4]. Furthermore, it may enable the exploration of new phenomena, including dynamics between a levitated nanomagnet and a single flux vortex [59-61], precession of a nonrotating magnet due to its intrinsic spin angular momentum [62], preparation of non-Gaussian quantum states [9], mechanically mediated quantum networks
$[3,63]$, detection of dark matter [58], and measuring the magnet's internal degrees of freedom [56].

This work was supported by the NSF, the Center for Ultracold Atoms (CUA), the ONR MURI Quantum OptoMechanics with Atoms and Nanostructured Diamond (QOMAND), the Vannever Bush Faculty Fellowship, and the Moore Foundation. This material is based upon work supported by the National Science Foundation Graduate Research Fellowship Program under Grants No. DGE1144152 and No. DGE1745303. Any opinions, findings, and conclusions or recommendations expressed in this material are those of the author(s) and do not necessarily reflect the views of the National Science Foundation. J. G. was supported by the European Union (SEQOO, H2020-MSCA-IF-2014, No. 655369). A. K. and A. S. were supported by the Department of Defense (DOD) through the National Defense Science and Engineering Graduate Fellowship (NDSEG) Program. C. G. B. was supported by the European Union (PWAQUTEC, H2020-MSCA-IF-2017 No. 796725). This work was performed in part at the Center for Nanoscale Systems (CNS), a member of the National Nanotechnology Coordinated Infrastructure Network (NNCI), which is supported by the National Science Foundation under NSF Grant No. 1541959. CNS is part of Harvard University. We gratefully acknowledge Frank Zhao for assistance with sample magnetization.

Note added.- Recently, we became aware of related work by Vinante et al. [64].

*jgieseler@fas.harvard.edu

Present address: ICFO-Institut de Ciencies Fotoniques, Mediterranean Technology Park, 08860 Castelldefels (Barcelona), Spain.

[1] P. Rabl, Cooling of mechanical motion with a two-level system: The high-temperature regime, Phys. Rev. B 82, 165320 (2010).

[2] M. J. A. Schuetz, G. Giedke, L. M. K. Vandersypen, and J. I. Cirac, High-fidelity hot gates for generic spin-resonator systems, Phys. Rev. A 95, 052335 (2017).

[3] P. Rabl, S. J. Kolkowitz, F. H. L. Koppens, J. G. E. Harris, P. Zoller, and M. D. Lukin, A quantum spin transducer based on nanoelectromechanical resonator arrays, Nat. Phys. 6, 602 (2010).

[4] S. Kolkowitz, A. C. Bleszynski Jayich, Q. P. Unterreithmeier, S. D. Bennett, P. Rabl, J. G. E. Harris, and M. D. Lukin, Coherent sensing of a mechanical resonator with a single-spin qubit, Science 335, 1603 (2012).

[5] S. D. Bennett, N. Y. Yao, J. Otterbach, P. Zoller, P. Rabl, and M. D. Lukin, Phonon-Induced Spin-Spin Interactions in Diamond Nanostructures: Application to Spin Squeezing, Phys. Rev. Lett. 110, 156402 (2013).

[6] M. S. J. Barson, P. Peddibhotla, P. Ovartchaiyapong, K. Ganesan, R. L. Taylor, M. Gebert, Z. Mielens, B. Koslowski, 
D. A. Simpson, L. P. McGuinness, J. McCallum, S. Prawer, S. Onoda, T. Ohshima, A. C. Bleszynski Jayich, F. Jelezko, N. B. Manson, and M. W. Doherty, Nanomechanical sensing using spins in diamond, Nano Lett. 17, 1496 (2017).

[7] J. van Wezel and T. H. Oosterkamp, A nanoscale experiment measuring gravity's role in breaking the unitarity of quantum dynamics, Proc. R. Soc. A 468, 35 (2012).

[8] W. Marshall, C. Simon, R. Penrose, and D. Bouwmeester, Towards Quantum Superpositions of a Mirror, Phys. Rev. Lett. 91, 130401 (2003).

[9] P. Rabl, P. Cappellaro, M. Dutt, L. Jiang, J. Maze, and M. D. Lukin, Strong magnetic coupling between an electronic spin qubit and a mechanical resonator, Phys. Rev. B 79, 041302 (2009).

[10] O. Arcizet, V. Jacques, A. Siria, P. Poncharal, P. Vincent, and S. Seidelin, A single nitrogen-vacancy defect coupled to a nanomechanical oscillator, Nat. Phys. 7, 879 (2011).

[11] B. Pigeau, S. Rohr, L. Mercier de Lépinay, A. Gloppe, V. Jacques, and O. Arcizet, Observation of a phononic Mollow triplet in a multimode hybrid spin-nanomechanical system, Nat. Commun. 6, 8603 (2015).

[12] F. Mintert and C. Wunderlich, Ion-Trap Quantum Logic Using Long-Wavelength Radiation, Phys. Rev. Lett. 87, 257904 (2001).

[13] S. Hong, M. S. Grinolds, P. Maletinsky, R. L. Walsworth, M. D. Lukin, and A. Yacoby, Coherent, mechanical control of a single electronic spin, Nano Lett. 12, 3920 (2012).

[14] O. Romero-Isart, M. L. Juan, R. Quidant, and J. Ignacio Cirac, Toward quantum superposition of living organisms, New J. Phys. 12, 033015 (2010).

[15] D. E. Chang, C. A. Regal, S. B. Papp, D. J. Wilson, J. Ye, O. Painter, H. Jeff Kimble, and P. Zoller, Cavity optomechanics using an optically levitated nanosphere, Proc. Natl. Acad. Sci. U.S.A. 107, 1005 (2010).

[16] T. Li, S. Kheifets, and M. Raizen, Millikelvin cooling of an optically trapped microsphere in vacuum, Nat. Phys. 7, 527 (2011).

[17] J. Gieseler, B. Deutsch, R. Quidant, and L. Novotny, Subkelvin Parametric Feedback Cooling of a Laser-Trapped Nanoparticle, Phys. Rev. Lett. 109, 103603 (2012).

[18] V. Jain, J. Gieseler, C. Moritz, C. Dellago, R. Quidant, and L. Novotny, Direct Measurement of Photon Recoil from a Levitated Nanoparticle, Phys. Rev. Lett. 116, 243601 (2016).

[19] O. Romero-Isart, L. Clemente, C. Navau, A. Sanchez, and J. I. Cirac, Quantum Magnetomechanics with Levitating Superconducting Microspheres, Phys. Rev. Lett. 109, 147205 (2012).

[20] M. Cirio, G. K. Brennen, and J. Twamley, Quantum Magnetomechanics: Ultrahigh-Q-Levitated Mechanical Oscillators, Phys. Rev. Lett. 109, 147206 (2012).

[21] M. W. Doherty, N. B. Manson, P. Delaney, F. Jelezko, J. Wrachtrup, and L. C. L. Hollenberg, The nitrogen-vacancy colour centre in diamond, Phys. Rep. 528, 1 (2013).

[22] J. M. Taylor, P. Cappellaro, L. Childress, L. Jiang, D. Budker, P. R. Hemmer, A. Yacoby, R. Walsworth, and M. D. Lukin, High-sensitivity diamond magnetometer with nanoscale resolution, Nat. Phys. 4, 810 (2008).

[23] J. R. Maze, P. L. Stanwix, J. S. Hodges, S. Hong, J. M. Taylor, P. Cappellaro, L. Jiang, M. V. Gurudev Dutt, E. Togan, A. S. Zibrov, A. Yacoby, R. L. Walsworth, and
M. D. Lukin, Nanoscale magnetic sensing with an individual electronic spin in diamond, Nature (London) 455, 644 (2008).

[24] S. Kolkowitz, A. Safira, A. A. High, R. C. Devlin, S. Choi, Q. P. Unterreithmeier, D. Patterson, A. S. Zibrov, V. E. Manucharyan, H. Park, and M. D. Lukin, Probing Johnson noise and ballistic transport in normal metals with a singlespin qubit, Science 347, 1129 (2015).

[25] B. Hensen, H. Bernien, A. E. Dréau, A. Reiserer, N. Kalb, M. S. Blok, J. Ruitenberg, R. F. L. Vermeulen, R. N. Schouten, C. Abellán, W. Amaya, V. Pruneri, M. W. Mitchell, M. Markham, D. J. Twitchen, D. Elkouss, S. Wehner, T. H. Taminiau, and R. Hanson, Loophole-free Bell inequality violation using electron spins separated by 1.3 kilometres, Nature (London) 526, 682 (2015).

[26] J. Druge, C. Jean, O. Laurent, M.-A. Méasson, and I. Favero, Damping and non-linearity of a levitating magnet in rotation above a superconductor, New J. Phys. 16, 075011 (2014).

[27] H. Barowski, K. M. Sattler, and W. Schoepe, Static and dynamic forces on a permanent magnet levitating between superconducting surfaces, J. Low Temp. Phys. 93, 85 (1993).

[28] V. V. Nemoshkalenko, E. H. Brandt, A. A. Kordyuk, and B. G. Nikitin, Dynamics of a permanent magnet levitating above a high-Tc superconductor, Physica (Amsterdam) 170C, 481 (1990).

[29] J. R. Hull and A. Cansiz, Vertical and lateral forces between a permanent magnet and a high-temperature superconductor, J. Appl. Phys. 86, 6396 (1999).

[30] T. Wang, S. Lourette, S. R. O'Kelley, M. Kayci, Y. B. Band, D. F. Jackson Kimball, A. O. Sushkov, and D. Budker, Dynamics of a Ferromagnetic Particle Levitated Over a Superconductor, Phys. Rev. Applied 11, 044041 (2019).

[31] L. P. Neukirch, J. Gieseler, R. Quidant, L. Novotny, and A. Nick Vamivakas, Observation of nitrogen vacancy photoluminescence from an optically levitated nanodiamond, Opt. Lett. 38, 2976 (2013).

[32] T. M. Hoang, J. Ahn, J. Bang, and T. Li, Electron spin control of optically levitated nanodiamonds in vacuum, Nat. Commun. 7, 1 (2016).

[33] A. Kuhlicke, A. W. Schell, J. Zoll, and O. Benson, Nitrogen vacancy center fluorescence from a submicron diamond cluster levitated in a linear quadrupole ion trap, Appl. Phys. Lett. 105, 073101 (2014).

[34] T. Delord, L. Nicolas, L. Schwab, and G. Hétet, Electron spin resonance from $\mathrm{NV}$ centers in diamonds levitating in an ion trap, New J. Phys. 19, 033031 (2017).

[35] I. Alda, J. Berthelot, R. A. Rica, and R. Quidant, Trapping and manipulation of individual nanoparticles in a planar Paul trap, Appl. Phys. Lett. 109, 163105 (2016).

[36] J.-F. Hsu, P. Ji, C. W. Lewandowski, and B. D’Urso, Cooling the motion of diamond nanocrystals in a magneto-gravitational trap in high vacuum, Sci. Rep. 6, 30125 (2016).

[37] M. C. O’Brien, S. Dunn, J. E. Downes, and J. Twamley, Magneto-mechanical trapping of micro-diamonds at low pressures, Appl. Phys. Lett. 114, 053103 (2019).

[38] See Supplemental Material at http://link.aps.org/ supplemental/10.1103/PhysRevLett.124.163604 for a derivation of the frozen dipole model and experimental details, which includes Refs. [24,39,40]. 
[39] A. A. Kordyuk, Magnetic levitation for hard superconductors, J. Appl. Phys. 83, 610 (1998).

[40] S. A. Beresnev, V. G. Chernyak, and G. A. Fomyagin, Motion of a spherical particle in a rarefied gas. Part 2. Drag and thermal polarization, J. Fluid Mech. 219, 405 (1990).

[41] P. Huillery, T. Delord, L. Nicolas, M. Van Den Bossche, M. Perdriat, and G. Hetet, Spin-mechanics with levitating ferromagnetic particles, Phys. Rev. B 101, 134415 (2020).

[42] R Skomski, Nanomagnetics, J. Phys. Condens. Matter 15, R841 (2003).

[43] R. A. Norte, J. P. Moura, and S. Gröblacher, Mechanical Resonators for Quantum Optomechanics Experiments at Room Temperature, Phys. Rev. Lett. 116, 147202 (2016).

[44] C. Reinhardt, T. Müller, A. Bourassa, and J. C. Sankey, Ultralow-Noise SiN Trampoline Resonators for Sensing and Optomechanics, Phys. Rev. X 6, 021001 (2016).

[45] Y. Tsaturyan, A. Barg, E. S. Polzik, and A. Schliesser, Ultracoherent nanomechanical resonators via soft clamping and dissipation dilution, Nat. Nanotechnol. 12, 776 (2017).

[46] A. H. Ghadimi, S. A. Fedorov, N. J. Engelsen, M. J. Bereyhi, R. Schilling, D. J. Wilson, and Tobias J. Kippenberg, Elastic strain engineering for ultralow mechanical dissipation, Science 360, 764 (2018).

[47] J. G. Longenecker, H. J. Mamin, A. W. Senko, L. Chen, C. T. Rettner, D. Rugar, and J. A. Marohn, High-gradient nanomagnets on cantilevers for sensitive detection of nuclear magnetic resonance, ACS Nano 6, 9637 (2012).

[48] R. Fischer, D. P. McNally, C. Reetz, and G. G. T. Assumpção, T. Knief, Y. Lin, and C.A. Regal, Spin detection with a micromechanical trampoline: Towards magnetic resonance microscopy harnessing cavity optomechanics, New J. Phys. 21, 043049 (2019).

[49] J.-P. Tetienne, L. Rondin, P. Spinicelli, M. Chipaux, T. Debuisschert, J.-F. Roch, and V. Jacques, Magnetic-fielddependent photodynamics of single NV defects in diamond: An application to qualitative all-optical magnetic imaging, New J. Phys. 14, 103033 (2012).

[50] L. Rondin, J.-P. Tetienne, T. Hingant, J.-F. Roch, P. Maletinsky, and V. Jacques, Magnetometry with nitrogenvacancy defects in diamond, Rep. Prog. Phys. 77, 056503 (2014).

[51] P. Forn-Díaz, L. Lamata, E. Rico, J. Kono, and E. Solano, Ultrastrong coupling regimes of light-matter interaction, Rev. Mod. Phys. 91, 025005 (2019).

[52] J. Gieseler, L. Novotny, and R. Quidant, Thermal nonlinearities in a nanomechanical oscillator, Nat. Phys. 9, 806 (2013).
[53] M. H. Abobeih, J. Cramer, M. A. Bakker, N. Kalb, M. Markham, D. J. Twitchen, and T. H. Taminiau, One-second coherence for a single electron spin coupled to a multiqubit nuclear-spin environment, Nat. Commun. 9, 2552 (2018).

[54] N. Bar-Gill, L. M. Pham, A. Jarmola, D. Budker, and R. L. Walsworth, Solid-state electronic spin coherence time approaching one second, Nat. Commun. 4, 1743 (2013).

[55] J. Prat-Camps, C. Teo, C. C. Rusconi, W. Wieczorek, and O. Romero-Isart, Ultrasensitive Inertial and Force Sensors with Diamagnetically Levitated Magnets, Phys. Rev. Applied 8, 034002 (2017).

[56] C. Gonzalez-Ballestero, J. Gieseler, and O. Romero-Isart, Quantum Acoustomechanics with a Micromagnet, Phys. Rev. Lett. 124, 093602 (2020).

[57] D. D. Bhaktavatsala Rao, S. Ali Momenzadeh, and J. Wrachtrup, Heralded Control of Mechanical Motion by Single Spins, Phys. Rev. Lett. 117, 077203 (2016).

[58] D. F. Jackson Kimball, A. O. Sushkov, and D. Budker, Precessing Ferromagnetic Needle Magnetometer, Phys. Rev. Lett. 116, 190801 (2016).

[59] L. Thiel, D. Rohner, M. Ganzhorn, P. Appel, E. Neu, B. Müller, R. Kleiner, D. Koelle, and P. Maletinsky, Quantitative nanoscale vortex imaging using a cryogenic quantum magnetometer, Nat. Nanotechnol. 11, 677 (2016).

[60] M. Pelliccione, A. Jenkins, P. Ovartchaiyapong, C. Reetz, E. Emmanouilidou, N. Ni, and A. C. Bleszynski Jayich, Scanned probe imaging of nanoscale magnetism at cryogenic temperatures with a single-spin quantum sensor, Nat. Nanotechnol. 11, 700 (2016).

[61] Y. Schlussel, T. Lenz, D. Rohner, Y. Bar-Haim, L. Bougas, D. Groswasser, M. Kieschnick, E. Rozenberg, L. Thiel, A. Waxman, J. Meijer, P. Maletinsky, D. Budker, and R. Folman, Wide-Field Imaging of Superconductor Vortices with Electron Spins in Diamond, Phys. Rev. Applied 10, 034032 (2018).

[62] C. C. Rusconi, V. Pöchhacker, K. Kustura, J. I. Cirac, and O. Romero-Isart, Quantum Spin Stabilized Magnetic Levitation, Phys. Rev. Lett. 119, 167202 (2017).

[63] C. C. Rusconi, M. J. A. Schuetz, J. Gieseler, M. D. Lukin, and O. Romero-Isart, Hybrid architecture for engineering magnonic quantum networks, Phys. Rev. A 100, 022343 (2019).

[64] A. Vinante, P. Falferi, G. Gasbarri, A. Setter, C. Timberlake, and $H$. Ulbricht, Ultralow mechanical damping with Meissner-levitated ferromagnetic microparticles, arXiv: 1912.12252. 